\title{
Cooperative action for molecular debromination reaction on $\mathrm{Cu}(110)$
}

Ana Barragán ${ }^{1,2}$, Ane Sarasola ${ }^{3,4}$, Lucia Vitali $^{1,2,5}$

${ }^{1}$ Centro de Fisica de Materiales CSIC UPV EHU, San Sebastian, Spain

${ }^{2}$ Departamento de Fisica de Materiales UPV, San Sebastian, Spain

${ }^{3}$ Departamento de Fisica Aplicada I UPV, San Sebastian, Spain

${ }^{4}$ Donostia International Physics Center DIPC, San Sebastian, Spain

${ }^{5}$ Ikerbasque, basque foundation for science, Bilbao, Spain

The metal-catalyzed coupling of halobenzene derivatives leading to biaryls and larger carbon-based structures is a fundamental reaction in chemical synthesis. Copper is the paradigmatic catalyzer of the Ullmann cross-coupling reaction. Despite this, its role in the reaction is still under debate. Here, we shed light on the mechanistic steps of the debromination, characterizing a prototypical molecule, namely 4,7-dibromobenzo[c]-1,2,5-thiadiazole (2Br-BTD), deposited on a Cu(110) surface.

By means of scanning probe techniques and first principle calculations, we demonstrate the oxidative addition of Cu atoms leading to a -C-Cu-Br metal-organic complex. The scission of the strongly bound bromine atoms requires the cooperative action of neighbouring complexes resulting in the formation of $\mathrm{Cu}$ coordinated BTD structures.

\section{References:}

1. A. Sarasola, A. Barragan, L. Vitali, J.Am.Chem.Soc. 140, 15631 (2018) 